\title{
Physicochemical properties and biocompatibility of a polymer-paclitaxel conjugate for cancer treatment
}

\section{Danbo Yang' \\ Sang $\operatorname{Van}^{2}$ \\ Jian Liu $^{2}$ \\ Jing Wang' \\ Xinguo Jiang ${ }^{3}$ \\ Yiting Wang' \\ Lei $\mathrm{Yu}^{1,2}$}

'Biomedical Engineering and Technology Institute, Institutes for Advanced Interdisciplinary Research, East China Normal University, Shanghai, China; 'Biomedical Group, Nitto Denko Technical Corporation, Oceanside, CA; ${ }^{3}$ School of Pharmacy, Fudan University, Shanghai, China
Correspondence: Lei Yu Nitto Denko Technical Corporation, 50 I Via Del Monte, Oceanside, CA 92058, USA

Tel + I 7604357026

Fax +I 7604357050

Email yu_lei@gg.nitto.co.jp
This article was published in the following Dove Press journal:

International Journal of Nanomedicine

20 October 2011

Number of times this article has been viewed

Background: Poly(L- $\gamma$-glutamylglutamine) paclitaxel (PGG-PTX) conjugate is a non-diblock polymeric drug nanoparticle intended to improve the therapeutic index of paclitaxel. The purpose of the present study was to elucidate further the physicochemical properties of PGG-PTX in order to proceed with its clinical development.

Methods and results: PGG-PTX was designed by integration of a hydrophobic paclitaxel conjugate through an added hydrophilic glutamic acid onto poly(L-glutamic acid). The addition of a flexible glutamic linker between PGA and paclitaxel resulted in spontaneous self-assembly of a PGG-PTX conjugate into nanoparticles. The PGG-PTX conjugate was stable as a lyophilized solid form. An in vitro viability experiment showed that PGG-PTX was effective after a longer incubation period, the same trend as Taxol. In vitro studies using NCI-H460 and B16F0 cancer cells demonstrated significantly high cellular uptake after 30 minutes of incubation. The in vivo biocompatibility of PGG-PTX conjugate was evaluated in the NCI-H460 tumor model, the assessment of tissue seemed to be normal after 21 days of treatment.

Conclusion: These results are encouraging for further development of non-block polymeric paclitaxel nanoparticles for treatment of cancer.

Keywords: polymer conjugate, paclitaxel, poly(L- $\gamma$-glutamylglutamine), drug delivery, physicochemical properties, nanoparticles

\section{Introduction}

Conventional small-molecule cancer chemotherapy has a low therapeutic index due to poor aqueous solubility, the inability to target tumor tissues selectively, and multidrug resistance. ${ }^{1}$ A number of technologies have been explored to increase water solubility and tumor selectivity, and to overcome the drug resistance of these anticancer drugs. Nanoparticulate technologies can be used to improve water solubility and bioavailability. ${ }^{2}$ Examples of such technologies include nanocrystal drug particles, nanocrystal/amorphous drugs stabilized with compatible carriers, micronanoparticulate/ water droplet drug stabilized by phospholipids, calcium phosphate-based nanoparticles, and injectable suspensions of nanoparticle albumin-bound technology, as well as microprecipitation, lipid emulsion, and disperse-phase technology. Polymer nanotherapeutics offer another route for improving water solubility, tumor selectivity, and drug resistance. ${ }^{3-5}$ A polymer-drug conjugate comprises three components, ie, a suitable polymeric carrier, a biodegradable linker, and a bioactive antitumor agent. A macromolecular polymeric conjugate is intended to prolong the circulation time by avoiding kidney filtration and to target tumors passively by the "enhanced permeability 
and retention" effect, attributed to discontinuous endothelium in the tumor vasculature and poor lymphatic tumor drainage. ${ }^{6}$

Like many other hydrophobic anticancer drugs, paclitaxel $l^{7}$ is hindered by poor bioavailability because of its low solubility in aqueous solution and lack of tumor selectivity. Nonetheless, paclitaxel formulated in a Cremophor $\mathrm{EL}^{\circledR}$ and ethanol mixture has been approved by the US Food and Drug Administration to treat patients with ovarian, breast, lung, and head and neck cancer, and advanced forms of Kaposi's sarcoma. The mixture of Cremophor $\mathrm{EL}$ and ethanol ${ }^{8}$ can solubilize paclitaxel at a $6 \mathrm{mg} / \mathrm{mL}$ concentration for infusion. Emulsification of paclitaxel has also been investigated using high-shear homogenization to improve its water solubility. ${ }^{9}$ Paclitaxel conjugated with a water-soluble polymer has recently been reported. Polymerpaclitaxel conjugates have been advanced in several clinical trials. ${ }^{10}$ The poly(L-glutamic acid)-paclitaxel conjugate (CT-2103) is considered the most advanced polymerpaclitaxel conjugate to date. ${ }^{4} \mathrm{CT}-2103$ has been investigated in Phase III trials, but has not been approved as yet by the Food and Drug Administration, ${ }^{11-13}$ so there is still a need for an efficacious polymer-paclitaxel conjugate for the treatment of cancer.

In an attempt to improve the pharmacological profile of paclitaxel, poly(L- $\gamma$-glutamylglutamine)-paclitaxel (PGGPTX) was designed as a polymer-drug conjugate. We have reported the preparation of PGG-PTX previously..$^{14}$ Poly(L$\gamma$-glutamylglutamine) contains double carboxyl groups for drug attachment as compared with one carboxyl group on the parent chain, polyglutamic acid, which provides adequate carrying capacity for paclitaxel, with a high probability of it being water-soluble. The resulting PGG-PTX has a macromolecular weight of approximately $80 \mathrm{kDa}$, which would be beneficial for retention in the circulation and for accumulation in tumors. ${ }^{15}$ Linkage of paclitaxel to the poly(L- $\gamma$-glutamylglutamine) backbone markedly alters its urinary and fecal excretion behavior. In a preclinical tumor growth regression study, PGG-PTX was administered intravenously via the tail vein in a mouse model of NCI-H460 carcinoma, 2008 human ovarian cancer, and B16F0, and the results showed that PGG-PTX outperformed protein-bound paclitaxel $\left(\right.$ Abraxane $\left.^{\circledR}\right)$ with respect to inhibition of tumor growth when both drugs were given at doses that produced similar degrees of acute weight loss. ${ }^{16}$ The purpose of the present study was to elucidate further the physicochemical properties of PGG-PTX in order to proceed with its clinical development. To this end, we confirmed the nanoparticle morphology of PGG-PTX by transmission electron microscopy (TEM) and investigated the long-term stability of lyophilized PGG-PTX at room temperature and at $4{ }^{\circ} \mathrm{C}$ by determining its particle size and release of free paclitaxel from PGG-PTX. We also compared its in vitro cytotoxicity in various cancer cell lines and studied its cellular uptake. Histology studies did not reveal any tissue irregularities in NCI-H460-bearing mice after administration of the maximum tolerated dose of PGG-PTX. The results showed that PGGPTX was a sufficiently stable and biocompatible non-diblock nanoparticulate prodrug. In this study, we presented novel data of cellular uptake of PGG-PTX and in vitro potency of five more cancer cell lines: C6 glioma, A549 lung, Hela kidney, Caco-2 colon, and HepG2 liver cancer cell lines. The novelty also included the assessment of in vivo tissue biocompatibility in the NCI-H460 tumor model.

\section{Materials and methods}

\section{Reagents and cell lines}

PGG-PTX was synthesized as described previously by our laboratory. ${ }^{14}$ Coumarin 6 was purchased from Sigma-Aldrich (St Louis, MO). Purified deionized water was prepared using the Milli-Q Plus system from Millipore Co (Bedford, MA). NCI-H460 cells were maintained in RPMI 1640 supplemented with $10 \%$ fetal bovine serum containing $100 \mathrm{U} / \mathrm{mL}$ penicillin and $100 \mu \mathrm{g} / \mathrm{mL}$ streptomycin. C6, A549, B16F0, and HepG2 cell lines were maintained in Dulbecco's modified Eagle's medium with the same supplements. The Caco- 2 cell line was grown in modified Eagle's medium supplemented with $10 \%$ fetal bovine serum, $100 \mathrm{U} / \mathrm{mL}$ penicillin, $100 \mu \mathrm{g} / \mathrm{mL}$ streptomycin, and $1 \%$ sodium pyruvate.

\section{Physicochemical properties of PGG-PTX PGG-PTX morphology}

The shape and surface morphology of PGG-PTX were investigated by TEM (JEM-2100, JEOL, Peabody, MA). A layer of the PGG-PTX polymer powder was obtained and deposited on a copper grid for TEM by application of $0.01 \%$ phosphotungstic acid after the PGG-PTX nanoparticle suspension was air-dried.

\section{Size measurements}

Samples of PGG-PTX were stored at $4^{\circ} \mathrm{C}$ and at room temperature for three months, and then tested for changes in particle size and size distribution within this time period. PGG-PTX sample was dissolved in phosphatebuffered solution ( $\mathrm{pH} 6.5$ ) and 45\% methyl alcohol buffer at a concentration of $2 \mathrm{mg} / \mathrm{mL}$. Particle size was measured 
using a Malvern Zetasizer Nano-ZS (Malvern Instruments, Worcestershire, UK). Analysis of each PGG-PTX analytical solution was performed in triplicate. An $850 \mu \mathrm{L}$ sample of solution was transferred to a Malvern disposable zeta cell with a $10 \mathrm{~mm}$ optical path for each measurement. For each PGG-PTX sample, the average volume diameter (nm) and polydispersity index were recorded. In addition, the experimental error for each parameter was recorded as the standard deviation.

\section{Stability experiments}

The same samples used for the size measurements were analyzed for free paclitaxel using size-exclusion high-pressure liquid chromatography methods to determine the stability of the polymer-paclitaxel conjugate. Chromatographic separations were carried out using a Shodex ${ }^{\circledR}$ OHpak SB-804 column $(30 \mathrm{~cm} \times 6.0 \mathrm{~mm}, 10 \mu \mathrm{m}$ particle size $)$ with a Shodex OHpak SB-G guard column $(5 \mathrm{~cm} \times 6.0 \mathrm{~mm}, 10 \mu \mathrm{m}$ particle size) from Showa Denko KK, Tokyo, Japan. The mobile phase was a solution of methanol-phosphate buffer ( $\mathrm{pH}$ 6.5, $45: 55, \mathrm{v} / \mathrm{v}$ ) at a flow rate of $0.525 \mathrm{~mL} /$ minute. The detection wavelength was carried out at $\lambda 228 \mathrm{~nm}$. The free amount of paclitaxel in the polymer was determined based on a standard curve for free paclitaxel.

\section{Thermogravimetric differential thermal analysis}

Thermal analyses were carried out using an SDT Q600 V8.3 Build 101 thermogravimetric differential thermal analyzer. The procedure was adapted from a previous published method. ${ }^{17}$ Briefly, known quantities of paclitaxel and PGGPTX were transferred to aluminum pans. The pans were crimped and weighed. Heat was applied between $30^{\circ} \mathrm{C}$ and $500^{\circ} \mathrm{C}$ at a scanning rate of $5^{\circ} \mathrm{C} /$ minute in a nitrogen atmosphere.

\section{Cell culture}

\section{Cellular uptake of coumarin 6-labeled PGG-PTX nanoparticles}

For visualizing the cellular uptake studies, coumarin 6-labeled PGG-PTX nanoparticles were prepared based on a published method ${ }^{18}$ with a minor modification of changing the polymer. Briefly, NCI-H460 cells were cultured on polylysine-coated glass cover slips. Aliquots of coumarin 6-loaded PGG-PTX nanoparticles $(1.0 \mathrm{mg} / \mathrm{mL})$ were dispersed on glass slides and covered with coverslips. The cells were washed three times with phosphate-buffered solution after incubation for 30 minutes and 4 hours. The nuclei were then counterstained by DAPI for 15 minutes.
Cellular uptake was visualized using a fluorescence microscope system (Olympus, Japan).

\section{Quantitative cellular uptake of coumarin 6-labeled PGG-PTX nanoparticles}

For quantitative cellular uptake studies, the coumarin 6-labeled PGG-PTX nanoparticles were dispersed in medium at concentrations of $1.25,2.5,5$, and $10 \mathrm{mg} / \mathrm{mL}$, and each concentration was incubated independently with NCI-H460 and $\mathrm{B} 16 \mathrm{~F} 0$ cells at $37^{\circ} \mathrm{C}$. The cells were washed three times with $100 \mu \mathrm{L}$ of phosphate-buffered solution after incubation for $0.5,1,2$, and 4 hours. Lysis buffer $(0.5 \%$ Triton X-100) was then added to the cells. Cell-associated fluorescence intensity from the coumarin 6-loaded nanoparticles was measured using a microplate reader (SpectraMax ${ }^{\circledR}$ M5) at an excitation wavelength of $430 \mathrm{~nm}$ and an emission wavelength of $485 \mathrm{~nm}$.

\section{In vitro cytotoxicity assay}

For cytotoxicity measurements, $100 \mu \mathrm{L}$ of cell suspension in culture medium at a concentration of $3 \times 10^{4}$ cells $/ \mathrm{mL}$ was seeded in each well of a 96 -well plate, and incubated at $37^{\circ} \mathrm{C}$ in a humidified atmosphere with $5 \% \mathrm{CO}_{2}$. After overnight incubation, serial dilutions of PGG-PTX were added to the plate. After further incubation for 48 or 72 hours, the cells were stained with standard MTS. Absorbance at $490 \mathrm{~nm}$ was measured with a microplate reader (Thermo Multiskan MK3). Cell viability was calculated as the absorbance in wells of treated cells normalized to controls, and the concentration of drug that inhibited cell viability by $50 \%$ $\left(\mathrm{IC}_{50}\right)$ was determined.

\section{Biocompatibility}

\section{Hemolysis test}

Hemolysis studies were carried out according to a published procedure. ${ }^{19}$ Briefly, red blood cells was freshly collected from a healthy male Sprague-Dawley rat and washed three times with $1 \times$ D-phosphate-buffered solution $(\mathrm{pH} 7.4)$ by centrifugation at $850 \mathrm{~g}$ for 15 minutes until the supernatant became clear. The red blood cells were adjusted to a density at $1 \times 10^{8}$ cells $/ \mathrm{mL}$ with $1 \times$ phosphate-buffered solution (pH 7.4) and an aliquot $(180 \mu \mathrm{L})$ of the suspension was added to each well of a round-bottom 96-well plate. Each testing material in phosphate-buffered solution $(20 \mu \mathrm{L})$ was added to each corresponding well. Triton X-100 (0.2\%) was used to generate $100 \%$ cell lysis as a positive control. The cells were incubated with the testing materials at $37^{\circ} \mathrm{C}$ for one hour on a three-dimensional rotation platform. After incubation, 
$100 \mu \mathrm{L}$ of supernatant was transferred to a new flat-bottom 96-well plate after centrifuge at $850 \mathrm{~g}$ for 15 minutes. The hemoglobin released from the lysed red blood cells was measured by spectrophotometry at $541 \mathrm{~nm}$. The data are presented as the mean of triplicate experiments ( $\%$ relative to $0.2 \%$ Triton $\mathrm{X}-100$ treatment). The remaining red blood cell pellet from the centrifugation was gently resuspended and red blood cell aggregation was monitored by microscopy.

\section{Histology study using hematoxylin and eosin staining}

To induce NCI-H460 tumors, nu/nu mice purchased from Vital River Laboratories (Beijing, China) were injected with $4 \times 10^{6} \mathrm{NCI}-\mathrm{H} 460$ cells in medium over each shoulder as previously reported. ${ }^{15}$ The nu/nu mice were randomly divided into two groups. One group comprised the experimental subjects, and the other group comprised controls. All the mice then received PGG-PTX at the maximum tolerated dose as described previously ${ }^{16}$ via tail vein injection when tumor size was about $100 \mathrm{~mm}^{3}$. After 21 days of treatment, various tissue samples were harvested. Tissue blocks were sectioned at a $5 \mu \mathrm{m}$ thickness and placed on plain slides. The slides were deparaffinized in xylene and rehydrated through graded alcohols. They were then stained in Harris hematoxylin for four minutes and rinsed in tap water for two minutes. Next, they were differentiated in $1 \%$ acid alcohol, rinsed in distilled $\mathrm{H}_{2} \mathrm{O}$, and stained blue in $1 \%$ ammonia alcohol. The slides<smiles>[R2]OC(=O)CCC(NC(=O)CCC(NC(C)(C)C(C)(C)C(=O)C(CCC(=O)O[Na])NC(C)(C)C)C(=O)O[Na])C(=O)O[Na]</smiles>

\section{PGG-PTX conjugate}

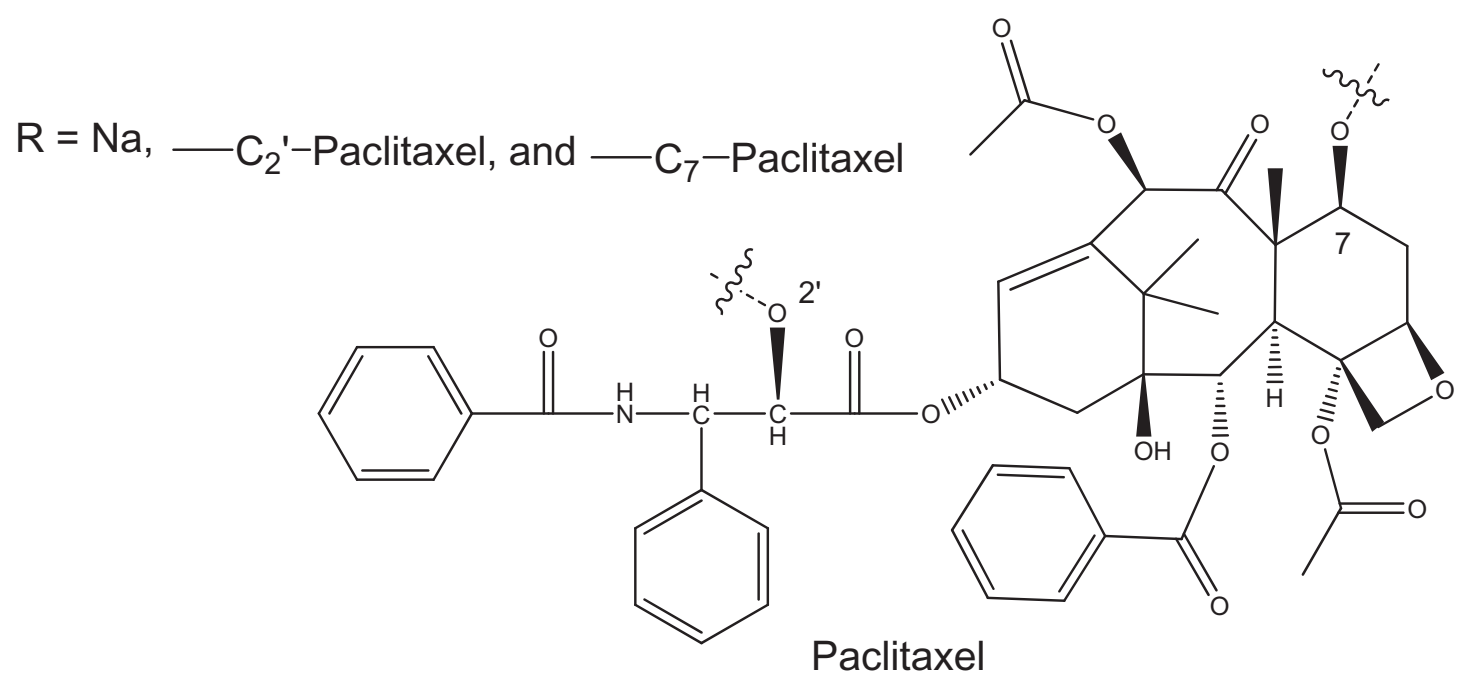

Figure I Structure of paclitaxel and PGG-paclitaxel conjugate. Abbreviations: PTX, paclitaxel; PGG, poly(L- $\gamma$-glutamylglutamine). 

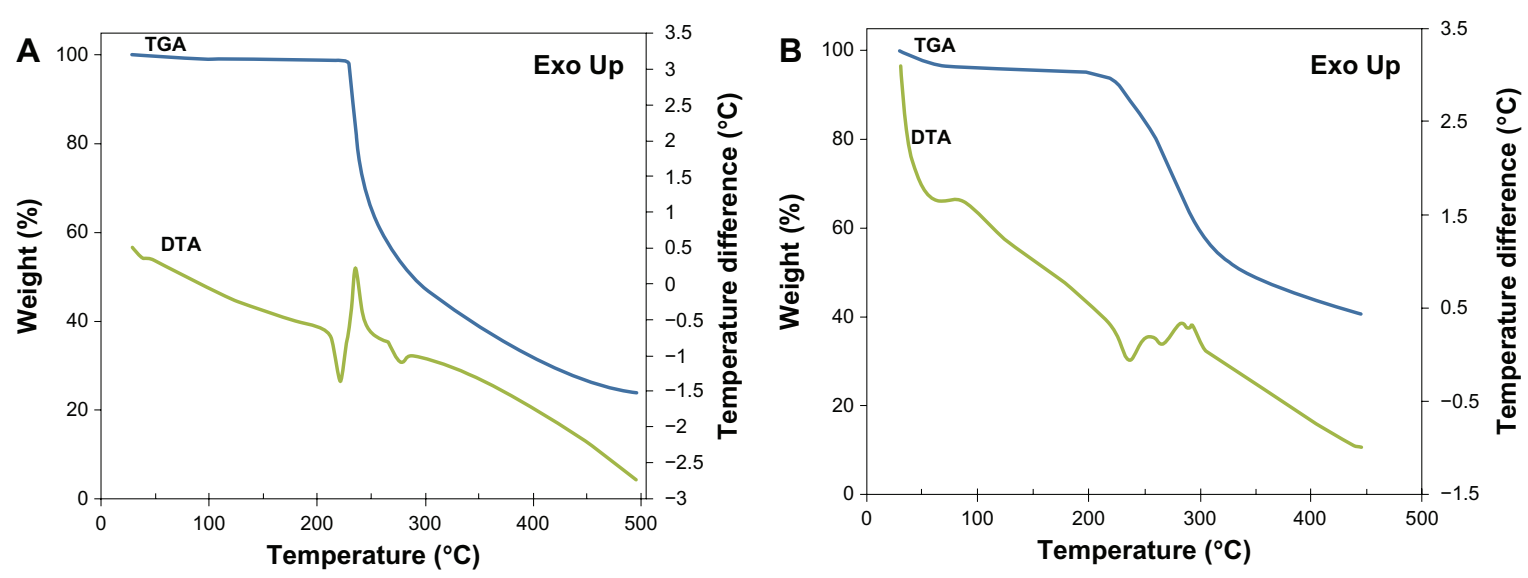

Figure 2 Thermogravimetric/differential thermal analyzer curve of free paclitaxel (A) and polymer PGG-PTX conjugate (B) with weight percent plotted against temperature. Abbreviations: PTX, paclitaxel; PGG, poly(l- $\gamma$-glutamylglutamine); TGA, thermogravimetric analysis; DTA, differential thermal analysis.

were then placed sequentially in $70 \%$ ethanol, $95 \%$ ethanol, eosin, $100 \%$ ethanol, and xylene for one minute each. The slides were mounted with Permount.

\section{Statistical analysis}

Data are expressed as the mean with $95 \%$ confidence intervals. Statistical tests were performed using the Student's $t$-test. The tests were considered to be statistically significant when $P$ values were less than 0.05 . All the statistical tests used the two-tailed method.

\section{Results and discussion}

The main aim of this study was to evaluate the physicochemical properties, in vitro potency, and in vivo tissue and blood biocompatibility of an anticancer PGG-PTX conjugate. Detailed study of its properties is essential if PGG-PTX is to proceed to clinical development. The PGGPTX conjugate was proposed because the conjugate should be able to improve the therapeutic index of paclitaxel. Previously, we have reported the synthesis of PGG-PTX and its in vitro cytotoxicity in a human lung NCI-460 cell line,,${ }^{14}$ its in vivo efficacy in a mouse model, ${ }^{16}$ and its superior pharmacokinetics and biodistribution in a human lung NCIH460 mouse model. ${ }^{15}$ In the present study, the physicochemical properties, morphology, and stability of PGG-PTX were examined. Subsequently, cell viability assays were performed to investigate the antiproliferative activity of the PGG-PTX conjugate in various cancer cell lines. Further, fluorescence microscopy assays were carried out for some of the cells pretreated with fluorescence-loaded carriers to elucidate the internalization capacity of the polymer drug delivery systems. Hemolysis and aggregation of PGG-PTX and poly(L- $\gamma$-glutamylglutamine) was investigated to determine their biocompatibility. Finally, the tissue histology study indicated that PGG-PTX did not have any detrimental effect on tissue integrity.

\section{Characterization of PGG-PTX conjugate}

PGG-PTX was synthesized as previously reported, ${ }^{14}$ and its structure is shown in Figure 1. The synthesis of PGG-PTX started with poly(L-glutamate) sodium salt coupled with glutamic acid, followed by conjugation with paclitaxel in the presence of 4-dimethylaminopyridine catalysis. This modification makes it possible to form stable nanoparticles of PGG-PTX alone without any additive and other polymer or lipid. The amphiphilic structure of PGG-PTX comprises hydrophobic paclitaxel and a hydrophilic polar carboxylate portion. Its bulky structure and large surface area makes it an excellent emulsifier, solubilizer, and functional

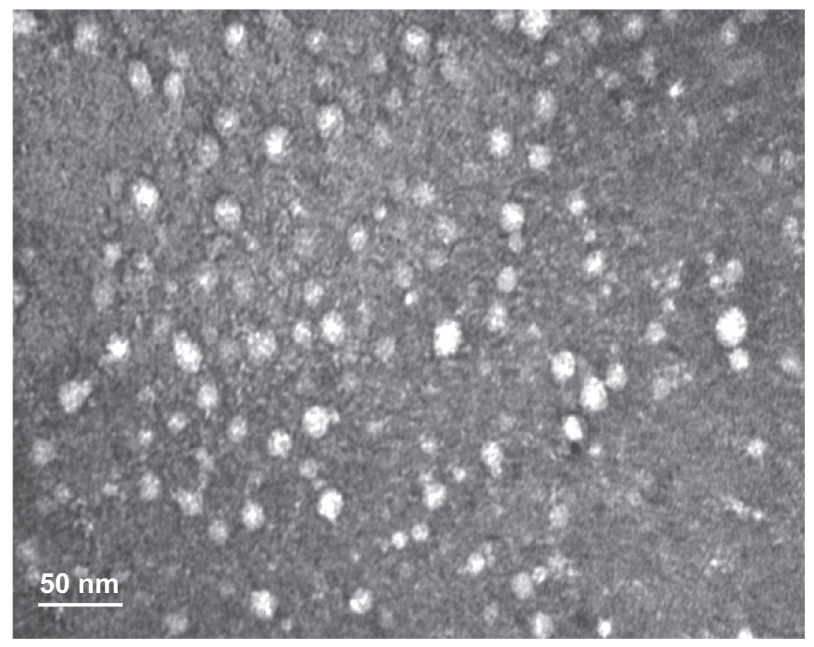

Figure 3 Transmission electron micrograph of poly(l- $\gamma$-glutamylglutamine)-paclitaxel nanoconjugates. 

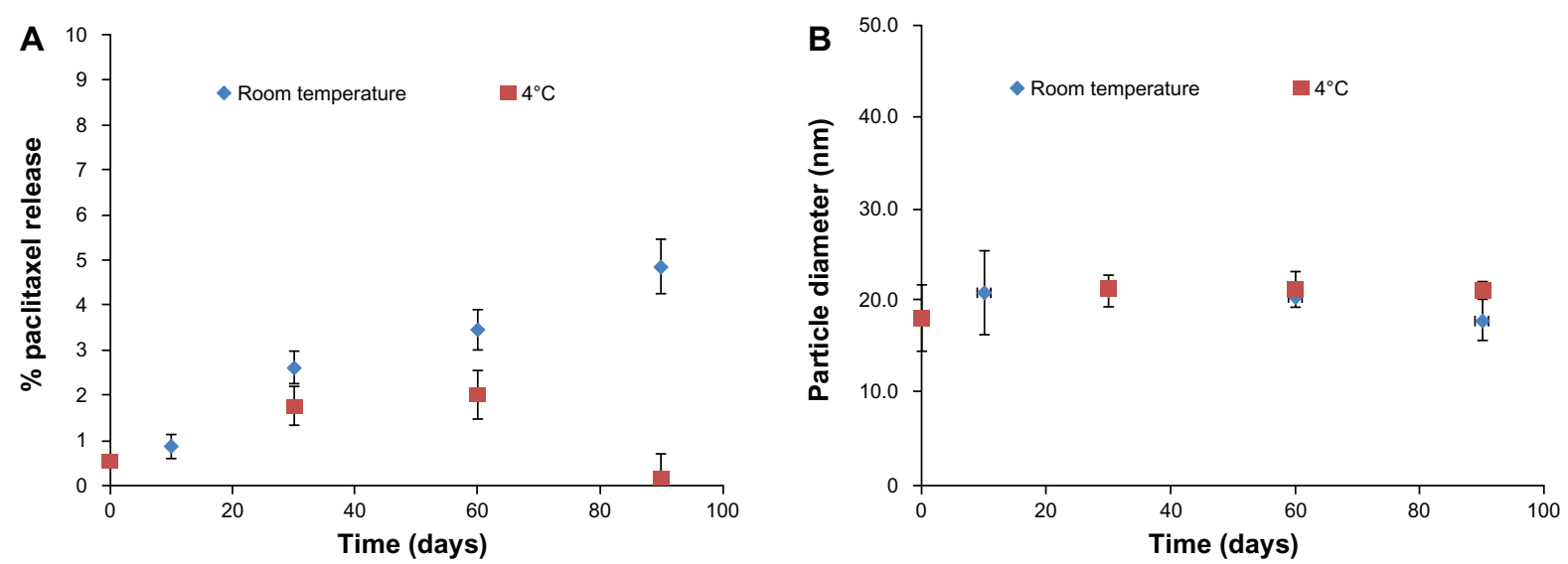

Figure 4 Stability of poly(l- $\gamma$-glutamylglutamine)-paclitaxel as a function of $(\mathbf{A})$ paclitaxel release and $(\mathbf{B})$ particle size at room temperature and $4^{\circ} \mathrm{C}$ over a period of three months. Note: Each point represents the mean of three samples with the standard error of the mean (vertical bars).

carrier for hydrophobic drugs. The paclitaxel-conjugated content of PGG-PTX was determined to be $36 \%$ (weight by weight) using an ultraviolet method. ${ }^{14,20}$ The ratios of the carboxyl groups to paclitaxel and the ratios of glutamic acid units to paclitaxel were calculated to be about 10:1 and $5: 1$, respectively, which might account for the high water solubility of the polymer $(>50 \mathrm{mg} / \mathrm{mL})$.

The thermal stability of the PGG-PTX polymer was identified by thermogravimetric analysis and differential thermal analysis. The initial mass of the material subjected to analysis was about $10.0 \mathrm{mg}$. The thermogravimetric analysis and differential thermal analysis curves are shown in Figure 2. The thermogravimetric analysis curve showed dehydration at $105^{\circ} \mathrm{C}$ and a moisture content of $11.7 \%$ gravimetrically evaluated for the PGG-PTX conjugate and $0.8 \%$ for free paclitaxel. This high water content for PGG-PTX results from the hygroscopic carboxylate group in the PGG backbone. Differential thermal analysis thermograms revealed one endothermic peak at $220^{\circ} \mathrm{C}$ and another exothermic peak at $236^{\circ} \mathrm{C}$ for free paclitaxel (Figure 2A). The former was the melting temperature, and the latter was assigned to decomposition. In the region between $210^{\circ} \mathrm{C}$ and $300^{\circ} \mathrm{C}$, the endothermic peak seen in differential thermal analysis (Figure 2B) can be ascribed to the absorption of energy for the breaking of bonds at the initial stage of decomposition. These blunt endothermic and exothermic peaks indicate an amorphous sample, which is consistent with the literature published for polymers like PLGA and PLA. ${ }^{21,22}$

PGG-PTX has previously demonstrated a capacity for self-assembly in aqueous solution, forming nanoparticles of average size distribution at about $20 \mathrm{~nm}$ by dynamic light scattering ${ }^{14}$ as well as by modeling. ${ }^{23}$ In this study, a TEM image of PGG-PTX provided conclusive evidence that PGG-PTX did indeed form nanoparticles, and the particle sizes seemed to be consistent with previously reported results for dynamic light scattering ${ }^{14}$ and TEM. ${ }^{24}$ As shown in Figure 3, the PGG-PTX nanoparticles were of a regular spherical shape, with a relatively homogeneous size distribution. In addition, the nanoparticle size of PGG-PTX in solution did not seem to have any significant effect with a change of $\mathrm{pH}$ from 7.1 to 5.5 or by time, for at least 24 hours at ambient temperature (data not shown). It is generally accepted that $20 \mathrm{~nm}$ nanoparticles would be ideal for evading the reticuloendothelial system and kidney

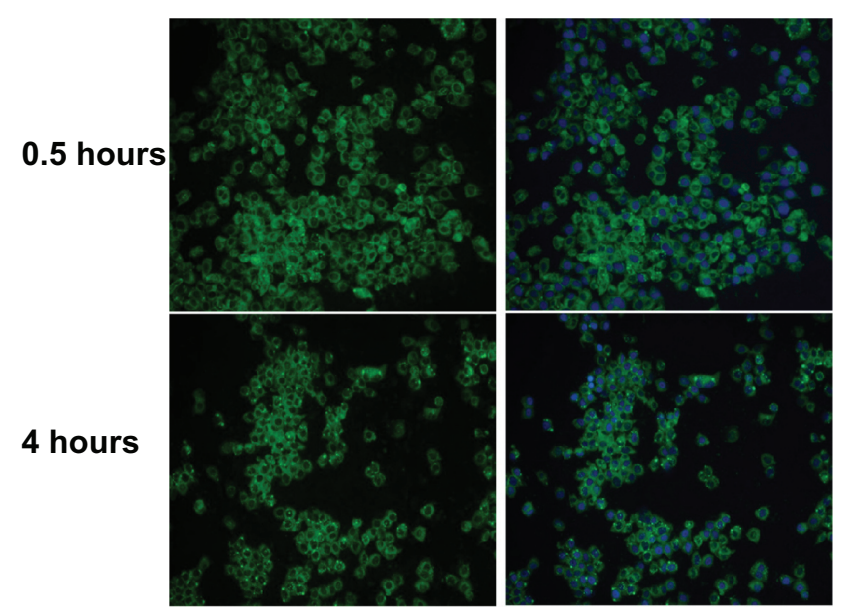

Figure 5 Fluorescence microscopy images showing internalization of $\mathrm{NCl}-\mathrm{H} 460$ cell treated with coumarin 6-labeled poly(l- $\gamma$-glutamylglutamine)-paclitaxel nanoparticles $\left(\mathrm{I} \mathrm{mg} / \mathrm{mL}\right.$ ) at $37^{\circ} \mathrm{C}$ for 30 minutes and 4 hours. On the left, the green-labeled corresponds to fluorescent poly(l- $\gamma$-glutamylglutamine)-paclitaxel loaded with coumarin 6. On the right, merged images of the nuclei stained with DAPI (blue) and of the cells uptake of coumarin 6-loaded poly(l- $\gamma$-glutamylglutamine)-paclitaxel (green). Notes: The results are representative of three independent experiments. Magnification 200x. 
A

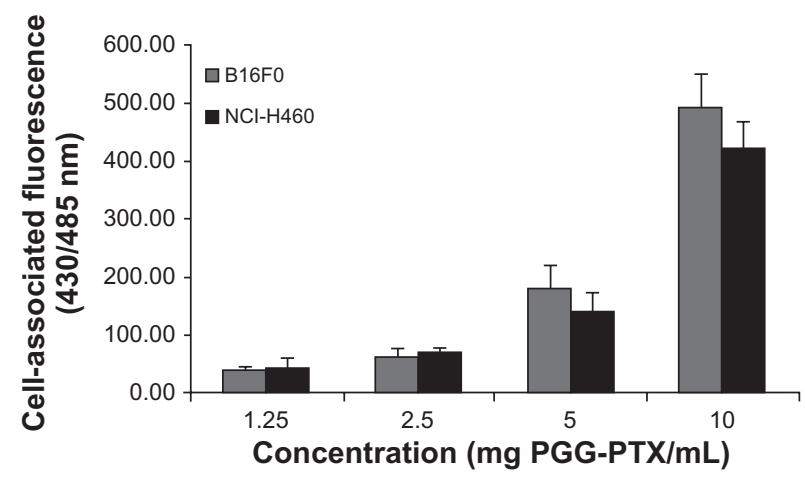

B

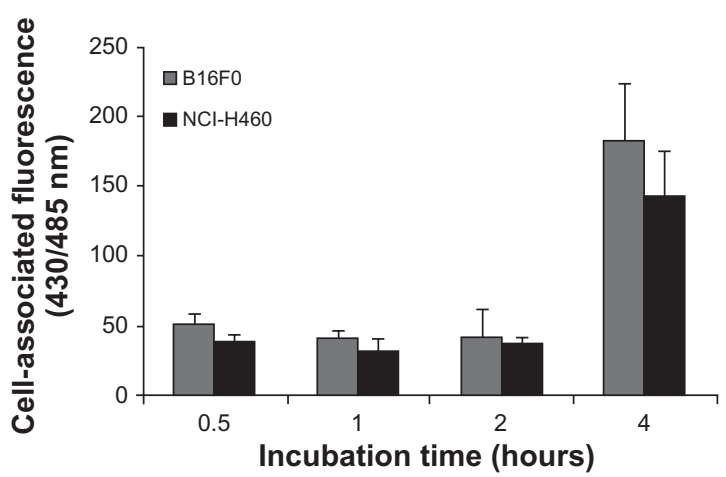

Figure 6 Cellular uptake of coumarin 6-loaded poly(l- $\gamma$-glutamylglutamine)-paclitaxel nanoparticles at (A) different concentrations incubated at $37^{\circ} \mathrm{C}$ for 4 hours, and (B) for different incubation periods of time at $5 \mathrm{mg}$ poly(l- $\gamma$-glutamylglutamine)-paclitaxel per $\mathrm{mL}$. Cell-associated fluorescence was measured at the excitation and emission wavelengths of 430 and $485 \mathrm{~nm}$, respectively.

Note: Results are expressed as the mean of three independent experiments \pm standard deviation $(n=3-4)$.

filtration, and might be effective carriers for drugs targeted to cancer tissues.

The physical stability of PGG-PTX in lyophilized form was evaluated at $4^{\circ} \mathrm{C}$ and room temperature to determine the stability of the ester linkage between the drug and polymer backbone by size exclusion high-pressure liquid chromatography and dynamic light scattering. A 90-day study concluded that lyophilized PGG-PTX could be stored for at least three months, with less than $2 \%$ and $5 \%$ release of free paclitaxel at $4^{\circ} \mathrm{C}$ and at room temperature, respectively (Figure 4A), without any significant change in its nanoparticle size in solution (Figure 4B). The same results were seen for PGG-PTX stored for two years at $4^{\circ} \mathrm{C}$ (data not shown). The stability of PGG-PTX as well as the high water solubility of its PGG backbone provides a good platform for poorly soluble aqueous drugs.

\section{In vitro cellular proliferation and uptake studies in tumor cell lines}

Clearly, the cellular uptake efficiency of a polymer-drug conjugate affects its therapeutic properties. To study the cellular uptake of nonfluorescent PGG-PTX, we adapted a published method ${ }^{18}$ by introducing green fluorescent coumarin 6 loaded onto PGG-PTX. Cellular internalization of coumarin 6-loaded PGG-PTX was examined in a model of human nonsmall cell lung cancer cells (NCI-H460). NCI-H460 cell lines were incubated with $5 \mathrm{mg} / \mathrm{mL}$ of the fluorescent coumarin 6-loaded PGG-PTX, under cell culture conditions for 30 minutes and 4 hours of incubation time. After washing, the cells with green fluorescence were visualized using a fluorescence microscope system, and their images were captured. Double fluorescence-labeling experiments of green fluorescence from coumarin 6 and blue fluorescence from
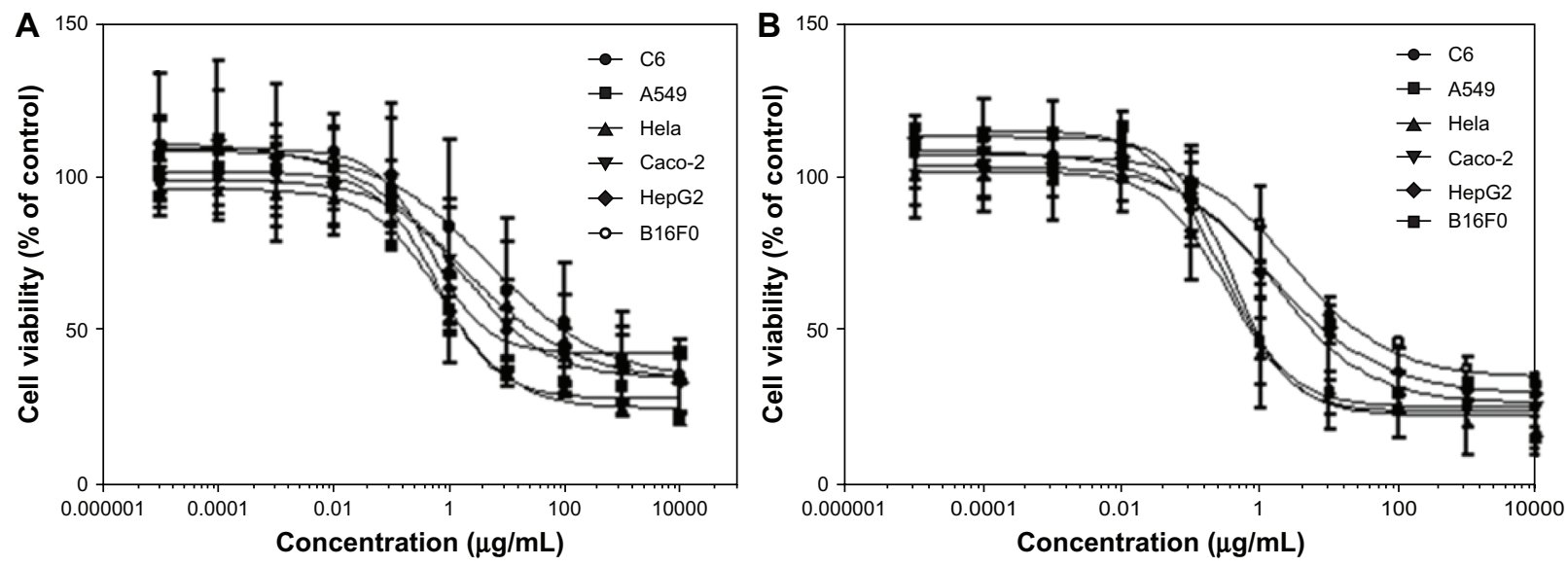

Figure 7 Cells were seeded in 96 -well cell culture plates overnight before exposure to poly(l- $\gamma$-glutamylglutamine)-paclitaxel at a concentration range of $0.0 \mathrm{I}-10 \mathrm{mg} / \mathrm{mL}$. Incubation continued for (A) 48 hours and (B) 72 hours before MTS staining. Survival of treated cells compared with untreated controls at each time point is shown. 
Table I IC I $_{50}(\mu \mathrm{g} / \mathrm{mL})$ of free paclitaxel and PGG-paclitaxel in various cell lines

\begin{tabular}{|c|c|c|c|c|}
\hline & \multicolumn{2}{|c|}{$\begin{array}{l}I_{50}(\mu g / m L) \text { value } \\
\text { of paclitaxel }\end{array}$} & \multicolumn{2}{|c|}{$\begin{array}{l}\mathrm{IC}_{50}(\mu \mathrm{g} / \mathrm{mL}, \mathrm{PTX} \text { equivalent) } \\
\text { value of PGG-PTX }\end{array}$} \\
\hline & 48 hours & 72 hours & 48 hours & 72 hours \\
\hline C6 & $.58 \pm 0.17$ & $0.08 \pm 0.02$ & $36.66 \pm 5.82$ & $1.14 \pm 0.10$ \\
\hline & $.12 \pm 0.02$ & $0.04 \pm 0.02$ & $8.28 \pm 2.82$ & 10 \\
\hline HeLa & & & & $1.91 \pm 0.87$ \\
\hline Caco-2 & $1.16 \pm 0.21$ & $0.92 \pm 0.40$ & $|82.04 \pm 53.6|$ & $13.73 \pm 4.68$ \\
\hline HepG2 & $1.26 \pm 0.45$ & $0.91 \pm 0.26$ & $420.98 \pm 136.96$ & $22.84 \pm 4.42$ \\
\hline BI6F0 & $3.82 \pm 1.77$ & $0.52 \pm 0.14$ & $217.18 \pm 57.86$ & $93.88 \pm|3.5|$ \\
\hline
\end{tabular}

Note: The IC $\mathrm{C}_{50}$ values are shown as a mean \pm standard deviation $(n=3)$. Abbreviations: PGG, poly(L- $\gamma$-glutamylglutamine); PTX, paclitaxel.

DAPI selectively labeling the nucleus were performed. As shown in Figure 5, the green fluorescent cells were observed after 30 minutes and 4 hours of incubation with the PGGPTX nanoparticles, which were seen to have penetrated the H460 cells. The images in the left column show the green fluorescence of the coumarin 6-loaded nanoparticles; the images in the right column show merged green fluorescence, and the blue fluorescence represents the nucleus stained by DAPI, which is circumscribed by green fluorescence representing the coumarin 6-loaded nanoparticles internalized in the cytoplasm. After 30 minutes, the coumarin 6-loaded PGG-PTX nanoparticles were clearly taken up by the $\mathrm{H} 460$ cells.

To evaluate quantitatively the cellular uptake and kinetic internalization of the PGG-PTX nanoparticles further, the effects of various concentrations of coumarin 6-labeled PGG-PTX nanoparticles and different incubation times were investigated in two cell lines, ie, NCI-H460 and B16F0. ${ }^{15}$ The results are shown in Figure 6. After 4 hours of incubation, a concentration-dependent increase in the cell-associated fluorescence intensity was observed in both cell lines (Figure 6A). There was no statistically significant differences in cell-associated fluorescence between the NCI-H460 and B16F0 cells $(P>0.05)$. Notably, no difference in fluorescent intensity was observed at a PGG-PTX concentration of $5 \mathrm{mg} / \mathrm{mL}$ (paclitaxel equivalent) in either cell line from 30 minutes to 2 hours (Figure 6B). The intensity of cell-associated fluorescence in the B16F0 cell line was 52.0, 41.9, and 41.9 for incubation times of 30 minutes, one hour, and 2 hours, respectively. A similar trend of fluorescent intensity was seen for the NCI-H460 cells, ie, 39.0, 32.1, and 37.8, corresponding to incubation times of 30 minutes, 1 hour, and 2 hours, respectively. However, a large increase in fluorescent intensity was observed between 2 and 4 hours of incubation time, ie, a 4.4-fold and 3.8-fold increase in fluorescent intensity for B16F0 and NCI-H460 cells, respectively. This unexpected increase in cellular uptake is still under investigation. The mechanism of in vitro cellular uptake of PGG-PTX nanoparticles can be assumed to be carrier-mediated endocytosis due to the small size of the particles. ${ }^{25}$

The in vitro cytotoxicity of PGG-PTX was investigated in various cancer cell lines at a concentration range of $0.01-10.0 \mathrm{mg} / \mathrm{mL}$ over 48 and 72 hours of incubation. The results are shown in Figure 7 and Table 1, and indicate that the concentration and duration of incubation of PGG-PTX has an impact on cell viability. For instance, the $\mathrm{IC}_{50}$ values of $36.6,8.2$, and $4.8 \mu \mathrm{g} / \mathrm{mL}$ for paclitaxel were equivalent to those of PGG-PTX determined after 48 hours of incubation with C6, A549 and HeLa cancer cells, whereas the $\mathrm{IC}_{50}$ values decreased significantly down to $1.1-1.9 \mu \mathrm{g} / \mathrm{mL}$ when PGG-PTX was incubated with the same cells for

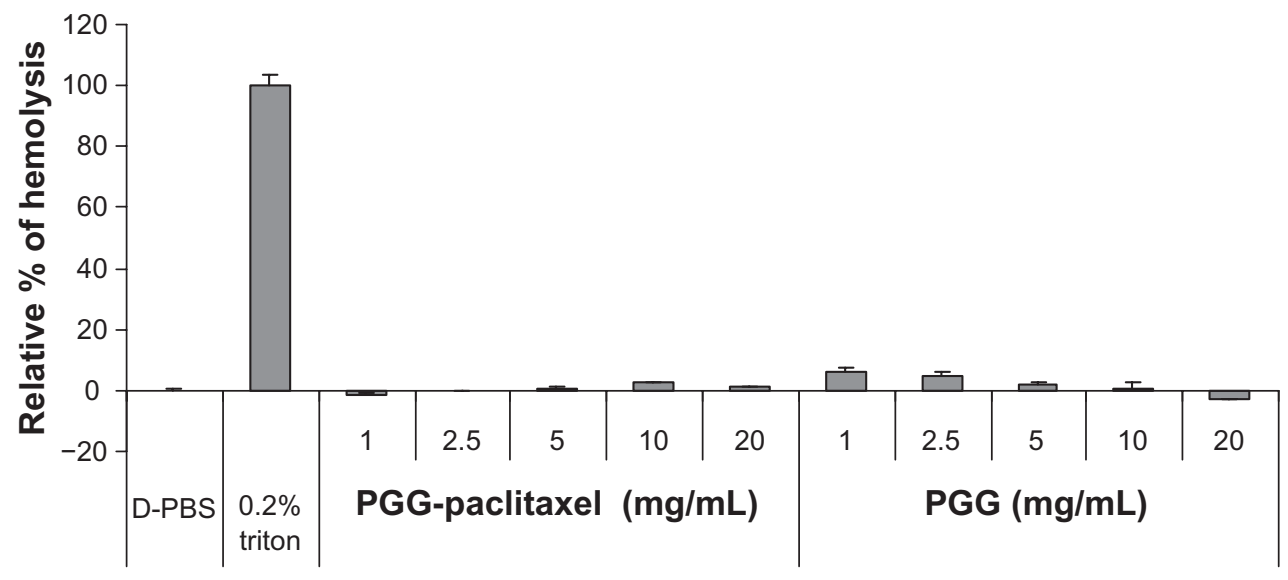

Figure 8 Effects of poly(L- $\gamma$-glutamylglutamine)-paclitaxel conjugate and poly(L- $\gamma$-glutamylglutamine) on red blood cell aggregation in pH 7.4 phosphate-buffered solution. Note: The relative percentages of hemolysis are shown as the mean \pm standard deviation $(n=3)$.

Abbreviations: PBS, phosphate-buffered solution; PGG, poly(L- $\gamma$-glutamylglutamine). 

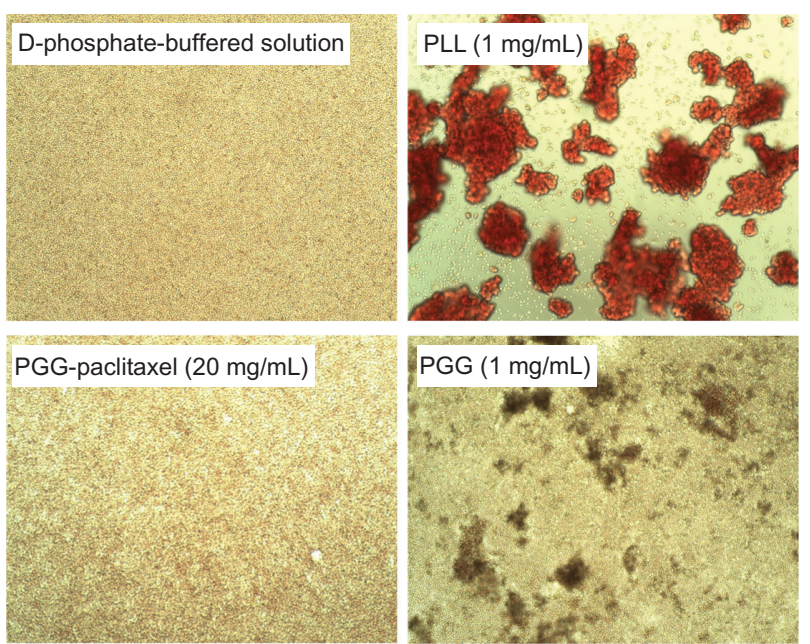

Figure 9 Effects of PGG-paclitaxel, PGG, and PLL on red blood cell aggregation in $\mathrm{pH} 7.4$ phosphate-buffered solution.

Note: The results are representative of three independent experiments.

Abbreviations: PGG, poly(L- $\gamma$-glutamylglutamine); PLL, poly(L-lysine).

72 hours. The longer incubation time achieving more potency might reflect the time needed for the active paclitaxel to be released from the polymer conjugate. Thus, use of the paclitaxel-conjugated compound for systemic delivery would be beneficial to avoid the toxicity associated with paclitaxel alone. In addition, it would take some time for the polymer-drug conjugate to reach tumor tissue, accumulate at the target site, and release active paclitaxel.

\section{Hemolysis and in vivo tissue biocompatibility}

Hemolysis was used to investigate the interaction between the PGG-PTX nanoconjugate and erythrocytes in buffer at $\mathrm{pH}$ 7.4. The results are shown in Figure 8, illustrating that PGG-PTX was not hemolytic under these testing conditions. PGG-PTX did not seem to interact with erythrocytes at up to $20 \mathrm{mg} / \mathrm{mL}$ of PGG-PTX in buffer at $\mathrm{pH}$ 7.4. However, the poly(L- $\gamma$-glutamylglutamine) backbone polymer seemed to aggregate with red blood cells even at a $1 \mathrm{mg} / \mathrm{mL}$ concentration of PGG-PTX (Figure 9). Furthermore, in order to verify whether the PGG-PTX conjugate impaired tissue function and morphology, the heart, liver, spleen, lung, kidney, brain, skin, and small intestine were evaluated for various microscopic changes after 21 days of treatment with PGG-PTX at a dose of $350 \mathrm{mg} / \mathrm{kg}$ via tail vein injection in tumor-bearing mice. As shown in Figure 10, all tissues seemed to be normal after 21 days of treatment with PGG-PTX when compared with the control group receiving saline (data not shown),
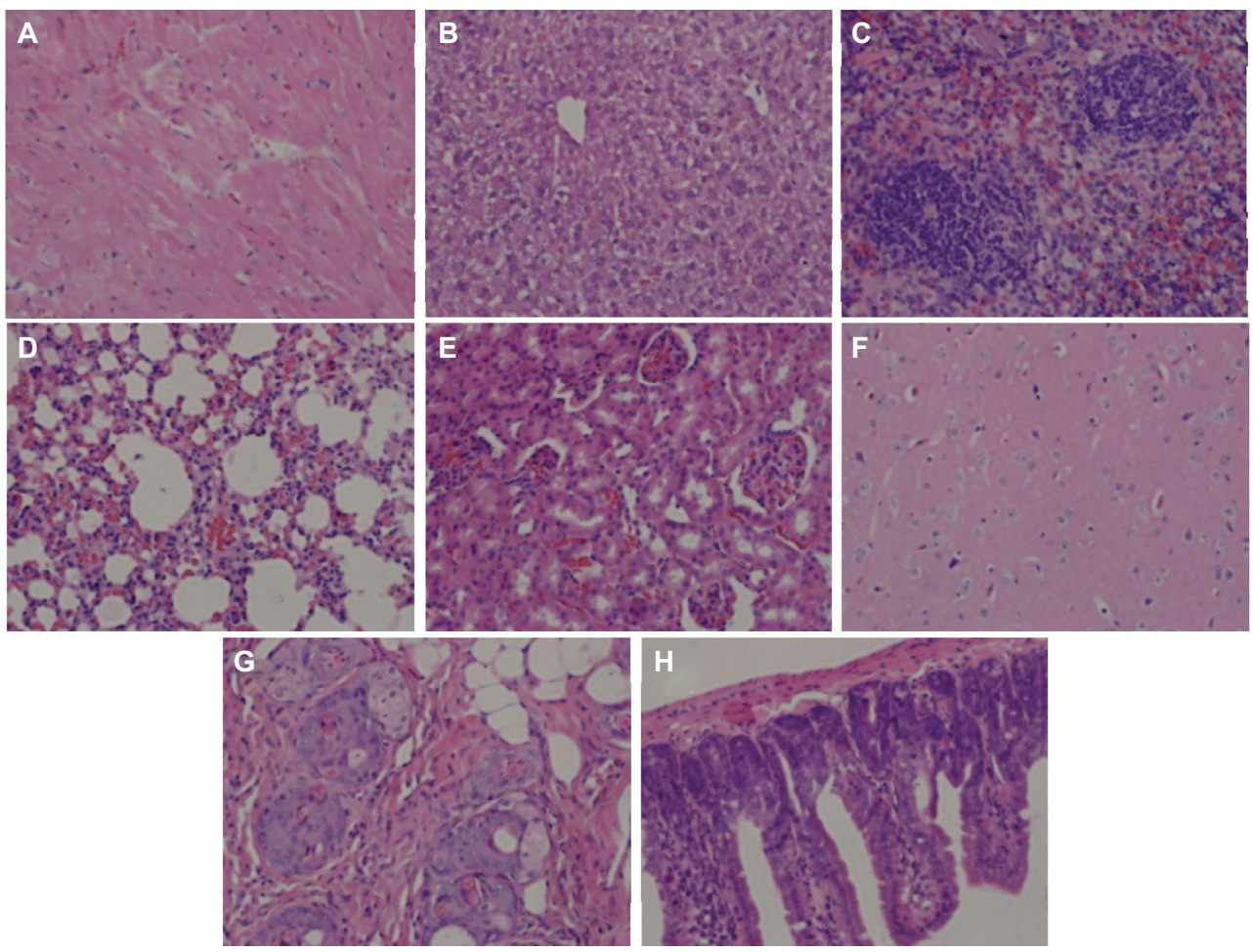

Figure $10 \mathrm{H} 460$ cells were injected into each shoulder of 6-8-week-old female athymic nude (nu/nu) mice. These mice were treated with a single intravenous injection of PGG-PTX $\left(350 \mathrm{mg} / \mathrm{mL}\right.$, paclitaxel equivalent) when tumor size reached an average volume of $100 \mathrm{~mm}^{3}$. Two mice were sacrificed after treatment, and sections of various tissues were examined by using hematoxylin and eosin staining. (A) heart, (B) liver, (C) spleen, (D) lung, (E) kidney, (F) brain, (G) skin, and (H) small intestine.

Note: Magnification I00x

Abbreviations: PGG, poly(L- $\gamma$-glutamylglutamine); PTX, paclitaxel. 
indicating that PGG-PTX is biocompatible and would not induce severe damage in patients after treatment.

\section{Conclusion}

Paclitaxel conjugated with a water-soluble polymeric carrier seems to be a promising strategy to enhance the physicochemical and biopharmaceutical properties of paclitaxel. The present PGG-PTX conjugate was characterized by high drug loading, high water solubility, long stability, and good biocompatibility. In view of the favorable physicochemical properties discussed in this paper, we are confident in advancing this conjugate to clinical development.

\section{Acknowledgments}

This work was supported by the National Basic Research Program of China (973 Program, 2007CB935802), the National Natural Science Foundation of China (60976004), the Shanghai Municipal Commission for Science and Technology (09JC1405300), and the Nitto Denko Technical Corporation.

\section{Disclosure}

The authors report no conflicts of interest in this work.

\section{References}

1. Moorthi C, Manavalan R, Kathiresan K. Nanotherapeutics to overcome conventional cancer chemotherapy limitations. J Pharm Pharm Sci. 2011;14:67-77.

2. Saffie-Siebert R, Ogden J, Parry-Billings M. Nanotechnology approaches to solving the problems of poorly water-soluble drugs. Drug Discov World Summer. 2005:71-76.

3. Yang D, Yu L, Van S. Clinically relevant anticancer polymer paclitaxel therapeutics. Cancers. 2011;3:17-42.

4. Haag R, Kratz F. Polymer therapeutics: concepts and applications. Angew Chem Int Ed Engl. 2006;45:1198-1215.

5. Li C, Price JE, Milas L, et al. Antitumor activity of poly(L-glutamic acid)-paclitaxel on syngeneic and xenografted tumors. Clin Cancer Res. 1999;5:891-897.

6. Maeda H, Wu J, Sawa T, Matsumura Y, Hori K. Tumor vascular permeability and the EPR effect in macromolecular therapeutics: a review. J Control Release. 2000;29:17-23.

7. Wani MC, Taylor HL, Wall ME, Coggon P, McPhail AT. Plant antitumor agents. VI. The isolation and structure of taxol, a novel antileukemic and antitumor agent from Taxus brevifolia. J Am Chem Soc. 1971;93: 2325-2327.

8. Sparreboom A, van Zuylen L, Brouwer E, et al. Cremophor ELmediated alteration of paclitaxel distribution in human blood: clinical pharmacokinetic implications. Cancer Res. 1999;59:1454-1457.

International Journal of Nanomedicine

\section{Publish your work in this journal}

The International Journal of Nanomedicine is an international, peerreviewed journal focusing on the application of nanotechnology in diagnostics, therapeutics, and drug delivery systems throughout the biomedical field. This journal is indexed on PubMed Central, MedLine, CAS, SciSearch ${ }^{\circledR}$, Current Contents ${ }^{\circledR} /$ Clinical Medicine,
9. Constantinides PP, Lambert KJ, Tustian AK, et al. Formulation development and antitumor activity of a filter-sterilizable emulsion of paclitaxel. Pharm Res. 2000;17:175-182.

10. Duncan R. The dawning era of polymer therapeutics. Nat Rev Drug Discov. 2003;2:347-360.

11. O’Brien MER, Socinski MA, Popovich AY, et al. Randomized Phase III trial comparing single-agent paclitaxel poliglumex (CT-2103, PPX) with single-agent gemcitabine or vinorelbine for the treatment of PS 2 patients with chemotherapy-naïve advanced non-small cell lung cancer. J Thorac Oncol. 2008;3:728-734.

12. Paz-Ares L, Ross H, O’Brien M, et al. Phase III trial comparing paclitaxel poliglumex vs docetaxel in the second-line treatment of non-small cell lung cancer. Br J Cancer. 2008;98:1608-1613.

13. Langer CJ, O'Byrne KJ, Socinski MA, et al. Phase III trial comparing paclitaxel poliglumex (CT-2103, PPX) in combination with carboplatin versus standard paclitaxel and carboplatin in the treatment of PS 2 patients with chemotherapy-naïve advanced non-small cell lung cancer. J Thorac Oncol. 2008;3:623-630.

14. Van S, Das SK, Wang X, et al. Synthesis, characterization, and biological evaluation of poly(1-g-glutamylglutamine)-paclitaxel nano-conjugate. Int J Nanomedicine. 2010;5:825-837.

15. Wang X, Zhao G, Van S, et al. Pharmacokinetics and tissue distribution of PGG-PTX, a novel macromolecular formulation of paclitaxel, in nu/ nu mice bearing NCI-460 lung cancer xenografts. Cancer Chemother Pharmacol. 2010;65:515-526.

16. Feng Z, Zhao G, Yu L, Gough D, Howell SB. Preclinical efficacy studies of a novel nano-particle-based formulation of paclitaxel that out-performs Abraxane. Cancer Chemother Pharmacol. 2010;65:923-930.

17. Panyam J, Sahoo SK, Prabha S, Bargar T, Labhasetwar V. Fluorescence and electron microscopy probes for cellular and tissue uptake of poly(D,L-lactide-co-glycolide) nanoparticles. Int J Pharm. 2003; 262:1-11.

18. Hu K, Li J, Shen Y, et al. Lactoferrin-conjugated PEG-PLA nanoparticles with improved brain delivery: in vitro and in vivo evaluations. J Control Release. 2009;134:55-61.

19. Yessine MA, Lafleur M, Meier C, Petereit HU, Leroux JC. Characterization of the membrane-destabilizing properties of different pH-sensitive methacrylic acid copolymers. Biochim Biophys Acta. 2003; 1613:28-38.

20. Li C, Yu DF, Newman RA, et al. Complete regression of well established tumors using a novel water-soluble poly(L-glutamic acid)-paclitaxel conjugate. Cancer Res. 1998;58:2404-2409.

21. Mainardes RM, Evangelista RC. PLGA nanoparticles containing praziquantel: effect of formulation variables on size distribution. Int $J$ Pharm. 2005;16:137-144.

22. Agrawal SK, Naomi S-D, Tew GN, Bhatia SR. Structural characterization of PLA-PEO-PLA solutions and hydrogels: crystalline vs amorphous PLA domains. Macromolecules. 2008;41:1774-1784.

23. Peng LX, Ivetac A, Chaudhari AS, et al. Characterization of a clinical polymer-drug conjugate using multiscale modeling. Biopolymers. 2010; 93:936-951.

24. Yang D, Van S, Jiang X, Yu L. Novel free paclitaxel-loaded poly (L- $\gamma$-glutamylglutamine)-paclitaxel nanoparticles. Int J Nanomedicine. 2011;6:85-91.

25. Ito J, Kato T, Kamio Y, et al. A cellular uptake of cis-platinumencapsulating liposome through endocytosis by human neuroblastoma cell. Neurochem Int. 1991;18:257-264.

\section{Dovepress}

Journal Citation Reports/Science Edition, EMBase, Scopus and the Elsevier Bibliographic databases. The manuscript management system is completely online and includes a very quick and fair peer-review system, which is all easy to use. Visit http://www.dovepress.com/ testimonials.php to read real quotes from published authors. 